\title{
Increasing Stroke Recognition and Behavioral Intent to Call Emergency Management Services in a Vietnamese American Population
}

\author{
Quyen Phan ${ }^{1^{*}}$, Gayle W Bentley ${ }^{2}$, Thomas V Joshua ${ }^{2}$ and Lovoria B Williams ${ }^{2,3}$ \\ ${ }^{1}$ Nell Hodgson School of Nursing, Emory University, 1520 Clifton Rd., Atlanta, GA, USA \\ ${ }^{2}$ Augusta University, College of Nursing 987 St. Sebastian Way, Augusta, GA, USA \\ ${ }^{3}$ University of Kentucky, College of Nursing, 751 Rose St., Lexington, KY, USA
}

"Corresponding author: Quyen Phan, Senior Clinical Instructor, Nell Hodgson Woodruff School Of Nursing, Emory University, 1520 Clifton Rd., Atlanta, GA 30322, Tel: 404-712-8558; E-mail: qphan@emory.edu

Received date: Feb 09, 2018; Accepted date: May 09, 2018; Published date: May 17, 2018

Copyright: $\odot 2018$ Phan Q, et al. This is an open-access article distributed under the terms of the Creative Commons Attribution License, which permits unrestricted use, distribution, and reproduction in any medium, provided the original author and source are credited.

\begin{abstract}
Objective: Serious complications of stroke, one of the leading causes of morbidity and mortality in the United States can be mitigated if treated in a timely manner. Stroke outcome disparity in Vietnamese Americans (VAs) is attributable to pre-hospital delay. To increase acute stroke recognition and behavior intent to call Emergency Management Services (EMS) among VAs and their families, an evidence-based, culturally and linguisticallyappropriate educational intervention was pilot-tested.
\end{abstract}

Methods: A convenience sample of 38 Vietnamese-speaking adults recruited from a free community clinic participated in a one-hour educational session. Pre and post intervention surveys were administered to assess knowledge and intent.

Results: Differences in pre-test and post-test means were large for stroke knowledge (Cohen's $D=2.39$ ), associated emergency action (Cohen's $D=2.57$ ) and behavioral intent to call EMS (Cohen's $D=0.94$ ).

Conclusion: A linguistically and culturally appropriate community-based education intervention may be effective in increasing stroke recognition and behavior intent to call EMS among VAs.

Keywords: Stroke; Behavior intent; Vietnamese American; Emergency action

\section{Introduction and Background}

Stroke is one of the leading causes of morbidity and mortality in the United States (US), and is a leading cause of serious long-term disability [1]. According to Yang et al. [2], strokes kills an estimated 140,000 people in US each year, and is the fifth leading cause of death. The risk and sequelae of stroke vary with race and ethnicity. Although Vietnamese Americans are not among the groups that are disproportionately affected by stroke incidence, they suffer the greatest proportionate mortality burden from the disease among the US Asian subgroup [3]. For example, the stroke proportionate mortality burden was 1.90 and 1.40 in Vietnamese women and men respectively, compared to 1.20 in non-Hispanic white women and 0.79 in nonHispanic white men [3].

Eighty-seven percent of strokes are ischemic which can be treated with tissue plasminogen activator (tPA) if eligible patients present to the hospital within three hours of symptom onset 1, [4]; however, most patients medically eligible for tPA treatment arrive outside of the $3 \mathrm{~h}$ treatment window. Stroke pre-hospital delays and high stroke mortality rates are particularly noted among ethnic minorities, with Vietnamese Americans having a $107 \%$ longer delay compared to non-Hispanic whites [3].
Interventions that have the greatest impact in reducing stroke delay are those that focus on early stroke recognition by the patient or the bystander. Therefore, to reduce stroke morbidity and subsequent mortality, both the Centers for Disease Control and Prevention (CDC) [5] and the American Stroke Association [4] recommend wide scale implementation of these interventions.

There is a dearth of evidence regarding prevention efforts to specifically target Vietnamese Americans. Cultural and language challenges might contribute to these lacks of prevention efforts, although literature is also sparse with these data. For example, Southeast Asian immigrants may be hesitant in Emergency Medical Services (EMS) utilization when experiencing stroke symptoms because of poor command of the English language. A qualitative study conducted within a Vietnamese American community in Philadelphia found that participants' beliefs that they had poor language skills led to their decision to seek care at a Vietnamese-speaking doctor's office or traditional healer rather than at a hospital or non-English speaking health care provider [6]. In the context of stroke, this health seeking behavior can further delay treatment, resulting in devastating complications or death.

Evidence suggests that effective interventions aimed at reducing pre-hospital stroke delay should: (1) focus on improving the population's stroke recognition [5]; (2) address the discrepancy between knowledge and action; (3) target the behavior of activating EMS $[7,8] ;$ (4) include culturally and linguistically-appropriate materials $[3,9] ;(5)$ involve the community in the planning process and 
be community-based [10]; and lastly (6) target high- risk individuals as well as family and bystanders [11]. Despite the high stroke mortality burden in the Vietnamese American population, there is a lack of research testing evidence-based interventions to reduce pre-hospital stroke delay in this population. The purpose of the study was to pilot test a linguistically and culturally appropriate educational intervention to improve stroke recognition and behavioral intent to call EMS, with the long-term goal of reducing pre-hospital stroke delay among Vietnamese Americans

\section{Methods}

\section{Design, setting, and sample}

The study had a one group pre-test/post-test design. The setting was a community-based organization that provides free health care services to uninsured Vietnamese Americans in a south eastern state. The organization, Boat People SOS (BPSOS), is a trusted national and local organization in the Vietnamese American community as it provides not only health services but other community empowerment services, such as computer and other educational classes [12].

The sample included thirty-eight Vietnamese American adults who met the following inclusion/exclusion criteria: 1) Vietnamese-speaking or bilingual and 2) 21 years of age and older. Because the educational intervention was presented using the Vietnamese language, those who did not speak or understand Vietnamese were excluded.

\section{Instruments}

The study involved three instruments that were translated from English into Vietnamese. Procedures used to translate the instruments are described later in this section.

\section{Demographic form}

Demographic data were collected on the following variables: age, gender, primary language spoken at home, education, history of stroke, and knowledge of someone else with stroke.

\section{Vignette questionnaire}

The sixteen-item vignette questionnaire was used to measure knowledge of stroke and appropriate action for stroke symptoms. It was developed and validated by Skolarus et al. [8] using a community based participatory research method. The questionnaire consists of 12 stroke and 4 non-stroke vignettes, each with a two-part question: The first part measures stroke symptom recognition (knowledge), while the second part measures appropriate action for stroke symptoms. Respondents are presented with 3 response options for knowledgeStroke, Not stroke, Don't know and 4 response options for action-Call doctor immediately, Wait a couple of hours, Call a family member immediately, or Call 911 immediately. Responses are scored as correct or not correct in relation to knowledge and action based on the scenario. Skolarus et al. [8] established content validity with experts and evaluated internal reliability using Cronbach $\alpha$, which was 0.88 for stroke knowledge and 0.83 for stroke action. Possible range of scores was zero to 16 for stroke knowledge, with higher scores indicating greater knowledge. Possible range of score for action was zero to 12 with higher scores indicating more appropriate stroke action.

\section{Behavior intent survey}

To measure the intent to call EMS for stroke symptoms which incorporated social norm, attitude, and self-efficacy constructs of the Theory of Planned Behavior (TPB), the author adapted a publicly available shortened seven-question survey (behavior intent survey) that Ajzen [13] designed. The possible range of the scores was from 1 to 5, with lower scores indicating greater intent to call EMS for stroke symptoms.

\section{Translation of study instruments}

To ensure the cultural and linguistic appropriateness of the study instruments, the author employed the community-engaged approach that is supported by several studies $[8,9]$. The author, who is native Vietnamese and fluent in the language, translated all the study instruments from English into Vietnamese. The trained translator staff of the intervention site BPSOS, subsequently reviewed the translated versions for linguistic and cultural appropriateness. An iterative process was utilized to incorporate the feedback from the translator into a revised version. The director of the community organization reviewed and approved the final versions of the instruments prior to use.

\section{Procedures}

The study was approved and a waiver of written consent were provided by the Augusta University Institutional Review Board. BPSOS clients were invited to attend one of four 45-min educational sessions. Prior to the intervention, clients were screened using the established inclusion/exclusion criteria. Participants then completed the demographic questionnaire, the vignette survey, and the behavioral intent questionnaire. No participant identifiers were collected; instead, participants were assigned unique numerical codes which were used to link pre and post-test surveys. Following completion of the educational intervention, participants completed the vignette survey and behavioral intent questionnaire again.

\section{Description of the intervention}

The intervention was based on Ajzen's Theory of Planned Behavior [13] and incorporated educational materials with demonstrated effectiveness. The author conducted the educational sessions in Vietnamese, using several teaching materials: The Face, Arm, Speech Time (FAST) message, illustrated in Figure 1. The FAST message, which visually depicts an individual experiencing stroke symptom with an emphasis on facial drooping, arm weakness, and slurred speech, has been shown to be reliable and valid in educating the public on stroke recognition and the importance of calling EMS [14]. The FAST message was presented in two formats: In a PowerPoint presentation and a refrigerator magnet distributed to participants; [2]. The 16vignette by Skolarus et al. [8] While the same 16 vignettes were used as a pre and post-questionnaire to measure stroke knowledge and intent to call EMS, the vignette questionnaire was also incorporated into the teaching materials. The teaching materials were used to (1) reinforce the stroke knowledge message, (2) provide the participants the context for stroke recognition, and (3) improve the participants' behavioral intent to call EMS. According to Ajzen [13], practicing a behavior improves self-efficacy, which directly impacts behavior intent. To improve self-efficacy, the participants practiced dialing 911 on a simulated telephone and saying a simple English phrase: "Help! Stroke!". After listening to the educational PowerPoint presentation 
Citation: Phan Q, Bentley GW, Joshua VT, Williams VB (2018) Increasing Stroke Recognition and Behavioral Intent to Call Emergency Management Services in a Vietnamese American Population. J Nurs Care 7: 459. doi:10.4172/2167-1168.1000458

Page 3 of 5

detailing the FAST components, reviewing the vignettes, and practicing dialing 911, the participants completed the postintervention questionnaires.

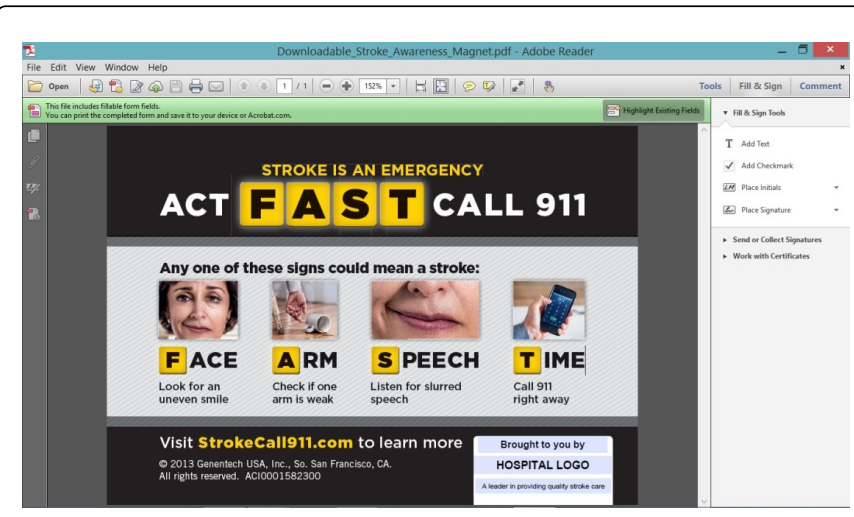

Figure 1: The Face, Arm, Speech Time (FAST) message.

\section{Data Analysis}

Descriptive statistics were generated to characterize the sample. Because of the small sample size, effect sizes were calculated for the differences in pre-test and post-test means using Cohen's D. Effect sizes were interpreted using Cohen's recommendation of $0.2=$ small effect, $0.5=$ medium effect, and $0.8=$ large effect [15].

\section{Results}

Thirty-eight Vietnamese-speaking adults participated in four different sessions. Demographic data for the sample are shown in Table 1. The majority (63\%) of the participants were aged 55 and above; most (55\%) were men. The majority (89\%) spoke Vietnamese only. Only one participant had a personal history of stroke, but the majority (58\%) knew someone who had experienced a stroke. Table 2 reports the pre and post test scores for stroke knowledge, stroke action, and intent to call EMS. The group sample sizes used to calculate Cohen's D varied due to missing data. Cohen's effect sizes were large for stroke knowledge, stroke action, and behavioral intent with the strongest effect on stroke knowledge and stroke action, and weakest related to behavioral intent.

\begin{tabular}{|c|c|c|}
\hline Characteristics & $\mathbf{n}$ & $\%$ \\
\hline \multicolumn{2}{|l|}{ Age in years } & \\
\hline $21-54$ & 14 & 36.8 \\
\hline 55 and older & 24 & 63.2 \\
\hline \multicolumn{3}{|l|}{ Gender } \\
\hline Male & 21 & 55.3 \\
\hline Female & 16 & 42.1 \\
\hline Missing & 1 & 2.6 \\
\hline \multicolumn{3}{|c|}{ Primary Language spoken at home } \\
\hline Vietnamese & 34 & 89.5 \\
\hline English & 4 & 10.5 \\
\hline \multicolumn{3}{|l|}{ Education } \\
\hline Grade 12 and above & 31 & 81.6 \\
\hline Up to Grade 11 & 7 & 18.4 \\
\hline \multicolumn{3}{|l|}{ History of Stroke } \\
\hline Yes & 1 & 2.6 \\
\hline No & 35 & 92.1 \\
\hline No Response & 2 & 5.3 \\
\hline \multicolumn{3}{|c|}{ Know someone with stroke } \\
\hline Yes & 22 & 57.9 \\
\hline No & 12 & 31.6 \\
\hline No Response & 4 & 10.5 \\
\hline
\end{tabular}

Table 1: Characteristics of Participants $(n=38)$.

\begin{tabular}{|l|l|l|l|l|l|l|l|}
\hline Variables & \multicolumn{2}{|l|}{ Pre- Intervention } & \multicolumn{2}{l|}{ Post- Intervention } & $\begin{array}{l}\text { Cohen's D effect } \\
\text { size }\end{array}$ \\
\hline & Mean & SD & Observed Range & Mean & SD & Range & \\
\hline Knowledge $(n=30)$ & 6.73 & 3.57 & $0-12$ & 13.56 & 1.88 & $7-15$ & 2.39 \\
\hline Stroke Action $(n=33)$ & 5.27 & 3.51 & $0-12$ & 11.75 & 0.61 & $9-12$ & 2.57 \\
\hline Intent $(n=33)$ & 3.95 & 0.96 & $01-5$ & 4.65 & 0.43 & $4-5$ & 0.94 \\
\hline
\end{tabular}

Table 2: Comparison of Pre-intervention and Post-Intervention Scores for Stroke Knowledge, Stroke Action, and Intent to Call EMS.

\section{Discussion}

To our knowledge, this study is the first to report a pilot test of an intervention targeting stroke recognition and intent to call EMS among a Vietnamese American population. Results suggested that the intervention holds promise as effective, due, in part, to the linguistic and cultural appropriateness of the tools, the author's fluency in the target population language and cultural practice, as well as the community-engaged approach. For example, the BPSOS staff provided input on decisions regarding the selection of the FAST message and the vignettes for inclusion in the intervention content over the use of other teaching materials. Not only did the author translate the teaching material into Vietnamese, but the staff at BPSOS provided feedback to ensure the accuracy and appropriateness of the translated materials. 
The FAST and vignette teaching materials were designed for the general population and an African American community, respectively, but they were deemed by the study investigators and BPSOS staff as most easy to adapt and made appropriate for use in the Vietnamese American population. Although widely used in US and Europe [16], the FAST message lacked the equivalent acronym in Vietnamese. The answer choices in the stroke action part of the vignettes, designed for an African American church-going community, did not include an action common in the Vietnamese American population-seeking help of an acupuncturist or traditional healer. However, the tools were simple to translate and most of the tools' elements were consistent with the Vietnamese American cultural practices such as family-related scenarios.

The intervention demonstrated that a brief educational session can be effective in increasing participants' knowledge of stroke signs and symptoms, stroke recognition, and behavior intent to call EMS for stroke signs and symptoms. The findings suggest that communitybased stroke education may have the potential to reduce pre-hospital delay in the Vietnamese American population. According to a systematic review by Bouckaert and colleagues [16], public campaigns resulted in increased stroke knowledge, but the duration of knowledge increase was short-lived. Future studies should include larger samples, a control group, and provide prolonged exposure to stroke education and include multimedia presentations to provide the population with sustained interventions.

As stroke victims' judgement and physical ability are often impaired, educating young bystanders and family members on stroke recognition and EMS activation may increase the likelihood of reducing prehospital delay. Morgenstern and colleagues [17] demonstrated in a randomized control trial study the effectiveness of educating middle school students to increase the likelihood of their detecting stroke symptoms in their loved ones and activating EMS accordingly. Results of their study indicated that focusing stroke education effort on young bystanders might improve stroke recognition and EMS activation. Given the high rate of smartphone use, particularly among young bystanders, the development of stroke awareness phone applications is also recommended. The translated stroke vignette and FAST message delivered via cell phone may be effective to educate the young Vietnamese American population about stroke recognition and the importance of timely EMS activation. Educating family members on stroke may be even more important among Vietnamese Americans because elders often live in multigenerational households.

\section{Limitations}

The main limitation of this study is the design lacked a control group and consequently, further testing of the intervention is warranted. The vignette tool, used to measure pre and post intervention stroke knowledge and action, also served as a key component of educational intervention which may have artificially increased scores for these variables at post-test. Some other limitations relate to the translation of the tool to Vietnamese. According to the Flesch-Kincaid Readability Grade, the vignette tool and the behavioral intent survey had appropriate health literacy readability levels of grades 5 and 5.9 respectively. However, due to the lack of a reading level appraising formula in Vietnamese, the reading level of the translated version could not be verified.

Another limitation of this study was the translated vignette tool needs more validity testing. Although all the social situations in the vignettes are culturally applicable to Vietnamese Americans, because the tool was designed for use and validated in the African American population, certain potential stroke actions such as visiting an acupuncturist or a traditional healer were not included on the tool.

\section{Implications for Practice}

A brief education intervention has the potential to positively impact stroke recognition and behavior intent to call EMS in the Vietnamese American population. Nurses play a pivotal role in patient education and disease prevention. Incorporating the FAST message and stroke vignettes into patient education, especially for high-risk stroke patients and their family, has the potential to reduce stroke delay time. Health care providers should stress the importance of calling EMS for stroke signs and symptoms rather than seeking care at a provider office, as this latter behavior contributes to the pre-hospital stroke treatment delay. Providers should also provide stroke education, utilizing existing tools such as video-players that are often available in patient waiting rooms. The stroke vignettes could easily be converted to multi-media presentations to be played for patients in waiting rooms [16]. Because the FAST message and stroke vignettes have shown effectiveness in improving stroke recognition and behavior intent to call EMS, nurses can utilize them as teaching materials. If time permits, role playing of the vignettes after FAST presentation might be more effective in improving participant's stroke self-efficacy, which directly influences the behavior intent to call EMS.

\section{Conclusions}

Pre-hospital stroke treatment delay in the ethnic minority community in general and the Vietnamese American community in particular could be reduced with culturally and linguistically appropriate community-based education. Nurses and health care providers can play a vital role in reducing pre-hospital stroke treatment delay if they target not only stroke recognition knowledge but also behavior intent to call EMS. Lastly, healthcare providers should include both high-risk stroke patients and potential bystanders in their stroke education.

\section{References}

1. Go AS, Mozaffarian D, Roger VL, Benjamin EJ, Berry JD, et al. (2014) Heart disease and stroke statistics-2014 update: a report from the American Heart Association. Circulation 129: e28-e292.

2. Yang Q, Tong X, Schieb L, Vaughan A, Gillespie C, et al. (2017) Vital signs: Recent trends in stroke death rates - United States, 2000-2015. MMWR 66: 933-939.

3. Jose PO, Frank AT, Kapphahn KI, Goldstein BA, Eggleston K, et al. (2014) Cardiovascular disease mortality in Asian Americans. J Am Coll Cardiol 64: 2486-2494.

4. Jauch EC, Saver JA, Adams HP, Bruno A, Connors JJ, et al. (2013) Guidelines for the early management of patients with acute ischemic stroke: A guideline for healthcare professionals from the American Heart Association/American Stroke Association. Stroke 44: 870-947.

5. Centers for Disease Control (2007) Prehospital and hospital delays after stroke onset - United States, 2005-2006. MMWR Morb Mortal Wkly Rep 56: 474-478.

6. Pham TM, Rosenthal MP, Diamond JJ (1999) Hypertension, cardiovascular disease, and health care dilemmas in the Philadelphia Vietnamese community. Fam Med 31: 647-651.

7. Teuschl Y, Brainin M (2010) Stroke education: discrepancies among factors influencing prehospital delay and stroke knowledge. Int J Stroke 5: 187-208. 
Citation: Phan Q, Bentley GW, Joshua VT, Williams VB (2018) Increasing Stroke Recognition and Behavioral Intent to Call Emergency Management Services in a Vietnamese American Population. J Nurs Care 7: 459. doi:10.4172/2167-1168.1000458

Page 5 of 5

8. Skolarus LE, Zimmerman MA, Murphy J, Brown DL, Kerber KA, et al (2011) Community-based participatory research: A new approach to engaging community members to rapidly call 911 for stroke. Stroke 42: 1862-1866.

9. Becker KJ, Fruin MS, Gooding TD, Tirschwell DL, Love PJ (2001) Community-based education improves stroke knowledge. Cerebrovasc Dis 11: 34-43

10. Willoughby DF, Sanders L, Privette A (2001) The impact of a stroke screening program. Public Health Nurs 18: 418-423.

11. Moser DK, Kimble LP, Alberts MJ, Alonzo A, Croft JB (2007) Reducing delay in seeking treatment by patients with acute coronary syndrome and stroke: a scientific statement from the American Heart Association Council on Cardiovascular Nursing and Stroke Council. Circulation 22: 326-343.

12. https://www.bpsos.org/bpsos---atlanta
13. Ajzen I (2006) Constructing a TPB questionnaire: conceptual and methodological considerations.

14. Payne GH, Fang J, Fogle CC, Oser CS, Wigand DA, et al. (2010) Stroke awareness: Surveillance, Educational Campaigns, and Public Health Practice. J Public Health Manag Pract 16: 345-358.

15. Cohen JW (1998) Statistical power analysis for the behavioral sciences (2nd edn). Lawrence Erlbaum Associates Hillsdale, NJ.

16. Bouckaert M, Lemmens R, Thijs V (2009) Reducing prehospital delay in acute stroke. Nat Rev Neurol 5: 477-483.

17. Morgenstern LB, Gonzales NR, Maddox KE (2007) A randomized, controlled trial to teach middle-school children to recognize stroke and call 911: The Kids Identifying and Defeating Stroke Project. Stroke 38: 2972-2978. 